\title{
Desplazamiento de un implante dental dentro del seno maxilar durante la segunda fase quirúrgica
}

\author{
GALLEGO MEDINA I * \\ SANCHEZ GARCES $\mathbf{M}^{\mathrm{a}} \mathbf{A} * *$ \\ BERINI AYTES L *** \\ GAY ESCODA C $* * * *$
}

\begin{abstract}
Gallego Medina I, Sánchez Garcés Ma A, Berini Aytés L, Gay Escoda C. Desplazamiento de un implante dental dentro del seno maxilar durante la segunda fase quirúrgica. Av Periodon Implantol. 2002; 14, 2: 81-88.
\end{abstract}

\begin{abstract}
RESUMEN
La rehabilitación bucal de los pacientes con edentulismo parcial o total con implantes dentales tiene amplias indicaciones que en ocasiones se ven limitadas por factores anatómicos y biológicos. Una de las localizaciones en la que frecuentemente puede existir una altura insuficiente de hueso alveolar, ya sea debido a la reabsorción ósea, a la excesiva neumatización del seno maxilar o a ambas causas, es la zona posterior del maxilar superior, lo que además suele ir acompañado de una baja calidad ósea. Todo esto hace que tanto la técnica quirúrgica convencional u otros procedimientos alternativos, como la elevación del seno maxilar, injertos en onlay, el uso de osteotomos y la osteotomía de Le Fort I, sean procedimientos quirúrgicos necesarios en muchas ocasiones para poder solucionar un caso de rehabilitación bucal con implantes. Por otra parte estas técnicas pueden alterar la anatomía y la fisiología del seno maxilar favoreciendo la aparición de complicaciones intra y postquirúrgicas. Una de las posibles complicaciones, aunque rara, es el desplazamiento del implante dentario hacia el seno maxilar durante la segunda fase quirúrgica, como es el caso que describimos en este artículo.
\end{abstract}

\section{PALABRAS CLAVE}

Implante dental, seno maxilar, desplazamiento, complicaciones.

\section{INTRODUCCIÓN}

En los artículos publicados sobre implantología dental podemos encontrar un pequeño porcentaje (alrededor del 10\%) de pacientes tratados con implantes que presentan complicaciones que llevan al fracaso del tratamiento $(1,2)$. Todo clínico experimentado que tiene un elevado número de casos exitosos, inevitablemente también tiene algunos casos con resultados dudosos. Algunos de estos resultados podrán etiquetarse como complicaciones y otros como fracasos (2).
Las complicaciones y los fracasos pueden clasificarse como: biológicos versus mecánicos, quirúrgicos versus protésicos, pero también admiten otras clasificaciones en función de diferentes factores.

La principal causa que conduce a una complicación o al fracaso de los implantes es la ausencia de un plan de tratamiento apropiado tanto quirúrgico como restaurador. En este sentido, una situación que puede conducir al fracaso es la colocación de implantes sin una clara comprensión del diseño de la prótesis final

* Odontóloga. Alumna del Master de Cirugía e Implantología Bucal. Facultad de Odontología de la Universidad de Barcelona.

** Profesora Asociada de Cirugía Bucal. Profesora del Master de Cirugía e Implantología Bucal. Facultad de Odontología de la Universidad de Barcelona.

*** Profesor Titular de Patología Quirúrgica Bucal y Maxilofacial. Profesor del Master de Cirugía e Implantología Bucal. Facultad de Odontología de la Universidad de Barcelona.

**** Catedrático de Patología Quirúrgica Bucal y Maxilofacial. Director del Master de Cirugía e Implantología Bucal. Facultad de Odontología de la Universidad de Barcelona. Cirujano Maxilofacial del Centro Médico Teknon. Barcelona. 


\section{AVANCES}

Volumen 14 - No2 - Julio 2002

y de la posición de los dientes, tanto por parte del odontólogo como del paciente. Así pues, es imprescindible un completo conocimiento anatómico y de los factores biológicos y biomecánicos para lograr el éxito del tratamiento (2).

Uno de los problemas que más frecuentemente nos encontramos es la ausencia de disponibilidad ósea. En el maxilar superior habitualmente la pérdida de molares y la posterior reabsorción ósea, no deja volumen óseo suficiente para la colocación de implantes, y además normalmente nos encontramos con una baja densidad ósea en la zona medular y una cortical muy delgada.

Para superar las limitaciones anatómicas tan frecuentes en el maxilar superior, existen numerosas técnicas quirúrgicas como por ejemplo la elevación del suelo del maxilar y la colocación de injerto de hueso autólogo en bloque o en partículas, de materiales de relleno o la mezcla de ambos. Esta técnica en concreto, se realiza cada vez con más frecuencia permitiendo así la colocación de implantes dentales en la región molar superior.

Actualmente con esta técnica ya se obtienen buenos resultados, aunque como es bien conocido, existen algunas complicaciones como la sinusitis maxilar, la reabsorción del injerto, la infección y el posible fallo del injerto, la pérdida de los implantes después de la primera fase quirúrgica y la fístula bucosinusal, en total, el porcentaje de aparición de complicaciones postoperatorias e incidencias debidas a la cirugía sinusal aplicada a la implantología es de un $8 \%$ (5). Otra posible situación es la perforación de la mucosa sinusal durante la preparación del lecho implantario, que para algunos autores se produce en el $35 \%$ de los casos $(3,4)$.

Otra posible complicación en la colocación de implantes en el maxilar superior es su posible desplazamiento hacia el interior del seno maxilar como el caso que a continuación describimos.

\section{CASO CLÍNICO}

Una paciente mujer de 38 años de edad, sin antecedentes patológicos de interés, fumadora de 30 cigarrillos al día, con edentulismo parcial y diagnosticada de periodontitis rápidamente progresiva fue atendida en el Máster de Cirugía e Implantología Bucal de la Universidad de Barcelona con el fin de realizar una rehabilitación bucal con prótesis implantosoportada. La paciente seguía tratamiento periodontal desde hacía un año y estaba en fase de mantenimiento con controles cada seis meses. En el plan de tratamiento

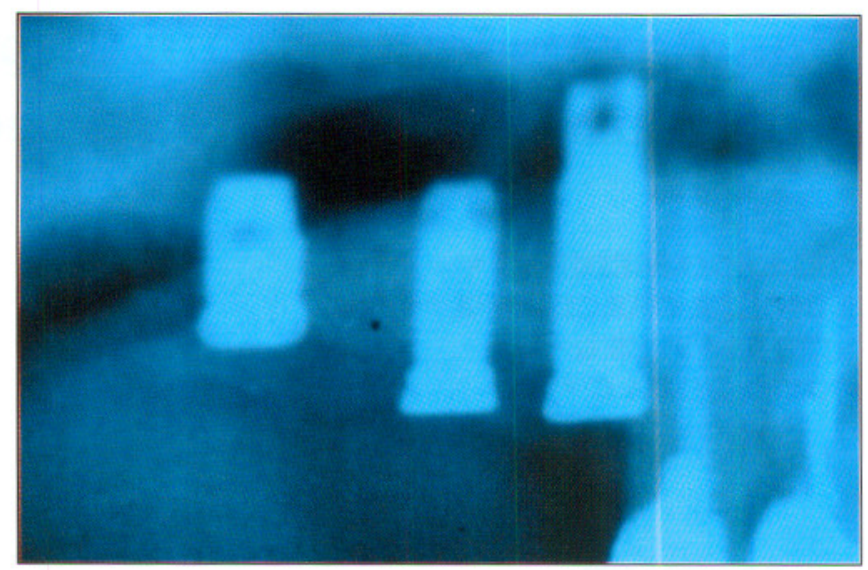

Fig. 1: Detalle de la ortopantomografía realizada posteriormente a la colocación de los implantes en posición 1.4, 1.5 y 1.6, donde se observa la reabsorción del hueso alveolar.

se decidió la extracción de los dientes 1.8 y 2.6, debido a que presentaban movilidad tipo III, y la colocación de seis implantes en las posiciones 1.6, 1.5, 1.4, $2.4,2.5$ y 2.6 .

En la primera cirugía se realizó la extracción del molar 1.8 y posteriormente la colocación de dos implantes sistema Nobel Biocare ${ }^{\oplus}$ estándar en posición 1.4 (4Ø x $15 \mathrm{~mm})$ y $1.5(4 \varnothing \times 10 \mathrm{~mm})$. También se colocó un tercer implante Implante Mk II en posición 1.6 (5Ø $7 \mathrm{~mm}$ ) (figura 1). Bajo premedicación antibiótica con $2 \mathrm{~g}$ de Amoxicilina una hora antes de la intervención, se realizó la cirugía con anestesia local (Articaína al 4\% con epinefrina 1:100.000). Se efectuó una incisión triangular con descarga mesial a nivel del canino 1.3 y no se precisaron técnicas de regeneración ósea guiada, aunque se obtuvo hueso autólogo, procedente del filtro de la aspiración, que fue colocado distalmente en el implante en posición 1.6. La estabilidad primaria de los implantes fue excelente y no hubo incidencias intraoperatorias. Se suturó con seda de $3 / 0$ atraumática C-16. Como tratamiento postoperatorio se le administró Amoxicilina 750 mg cada 8 horas durante una semana, Paracetamol $300 \mathrm{mg}$ con codeína fosfato $14.05 \mathrm{mg}$ cada 6 horas y Diclofenaco $100 \mathrm{mg}$ cada 12 horas ambos durante 4 días y se recomendaron enjuagues con un colutorio de diglucuronato de clorhexidina al $0,2 \%$ después de cada comida durante 15 días.

Es de destacar que la reabsorción ósea del maxilar superior derecho era intermedia entre la del tipo B y C según que clasificación; la calidad ósea era del tipo 3 según quien y durante la cirugía se observó una buena vascularización del tejido óseo y del colgajo.

Cuatro meses más tarde se colocaron tres implantes Mk II en el maxilar superior izquierdo, en las 


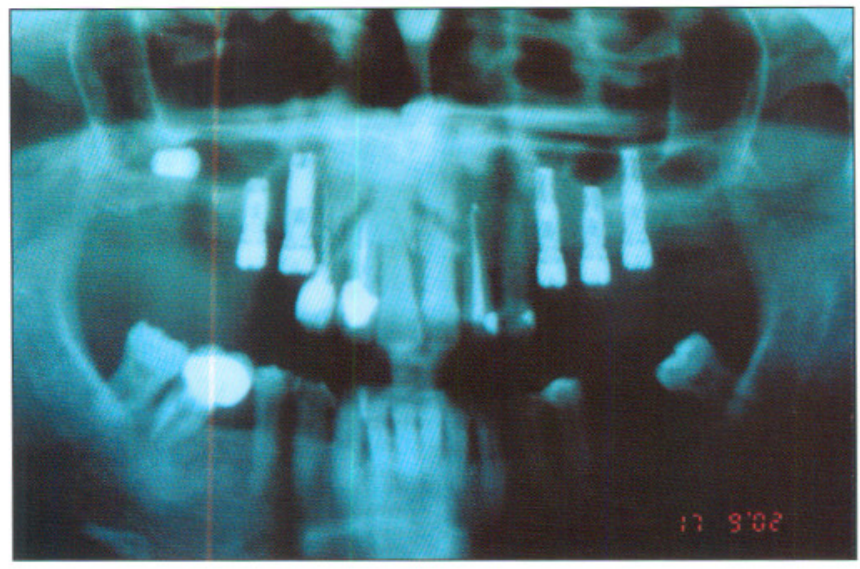

Fig. 2: Ortopantomografía donde se localiza el implante en posición 1.6 en el suelo del seno maxilar.

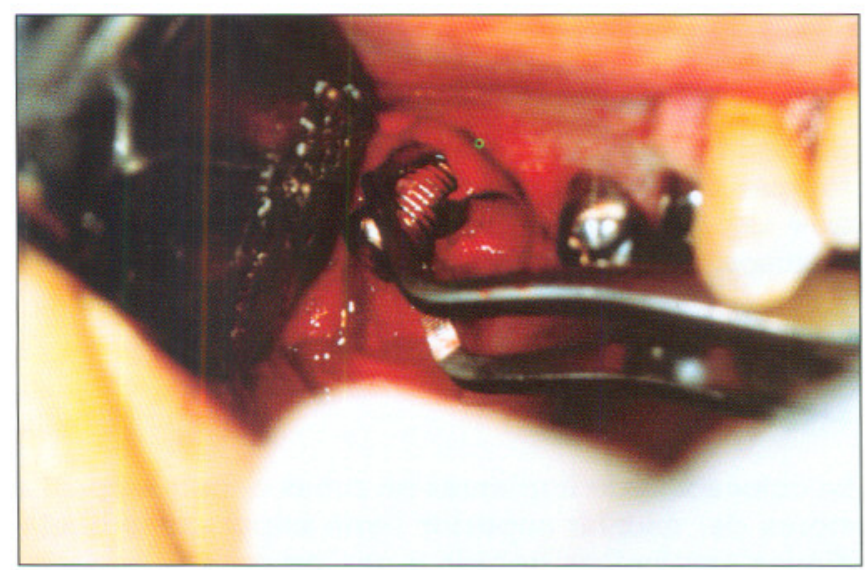

Fig. 4: Imagen intraoperatoria del momento en que se extrajo el implante desplazado al seno maxilar.

posiciones $2.4(3.75 \varnothing \times 15 \mathrm{~mm}), 2.5(3.75 \varnothing \times 11.5$ $\mathrm{mm})$ y $2.6(3.75 \varnothing \times 15 \mathrm{~mm})$ sin incidencias intraoperatorias. La medicación postoperatoria fue la misma que la pautada en la intervención quirúrgica anterior.

Durante los meses siguientes se observó la exposición de los tornillos de cierre de los implantes del maxilar superior derecho.

A los seis meses de la segunda intervención se realizó la segunda fase de todos los implantes colocados en el maxilar superior y se colocaron pilares de cicatrización de $4 \mathrm{~mm}$ de altura.

Durante la maniobra de desatornillado del tornillo de cierre del implante localizado en la posición 1.6 se produjo el desplazamiento de éste hacia el interior del seno maxilar derecho. Se interrumpió la cirugía para efectuar una ortopantomografía a la paciente en la que se pudo observar que el implante localizado en posición 1.6 se encontraba en el suelo del seno maxi-

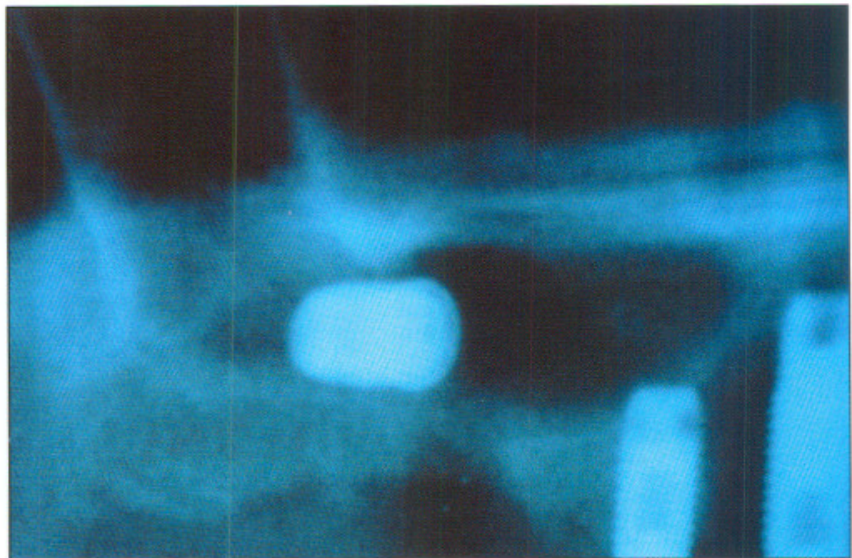

Fig. 3: Detalle de la ortopantomografía. Podemos observar una gran reabsorción del hueso alveolar y el implante en el interior del seno maxilar.

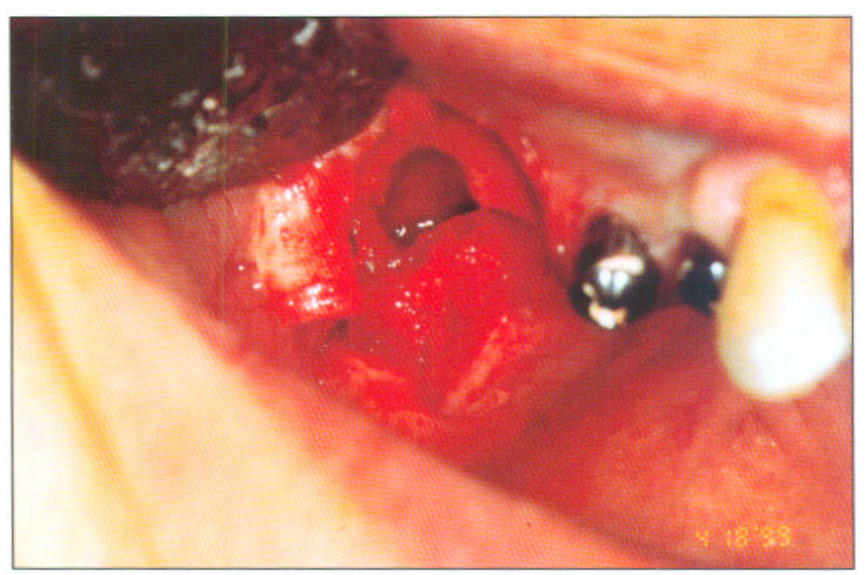

Fig. 5: En esta imagen podemos observar el gran defecto que quedó tras la remoción del implante, que comunicaba con el seno maxilar.

lar, en posición horizontal, por lo que se decidió realizar su extracción (figuras 2 y 3 ).

Bajo anestesia local con Articaina al 4\% y epinefrina 1:100.000 se realizó una incisión trapezoidal en el primer cuadrante preservando la encía vestibular de los otros dos implantes más mesiales ya descubiertos. Después de despegar el colgajo mucoperióstico se realizó una ostectomía antral de $1 x \mathrm{l} \mathrm{cm}$ en la zona correspondiente al lecho del implante desplazado previamente localizado en la ortopantomografía. A través de esta ventana se procedió al levantamiento de la mucosa del seno maxilar, se localizó el implante y se extrajo con unas pinzas acodadas (figuras 4,5 y 6).

Después de la extracción del implante migrado al seno maxilar se colocó una membrana reabsorbible, Bio-Guide, que cubría el defecto realizado por la ostectomía y el lecho del implante (figura 7) para prevenir una posible comunicación bucosinusal, se liberó el colgajo para facilitar su reposición sin tensión y 


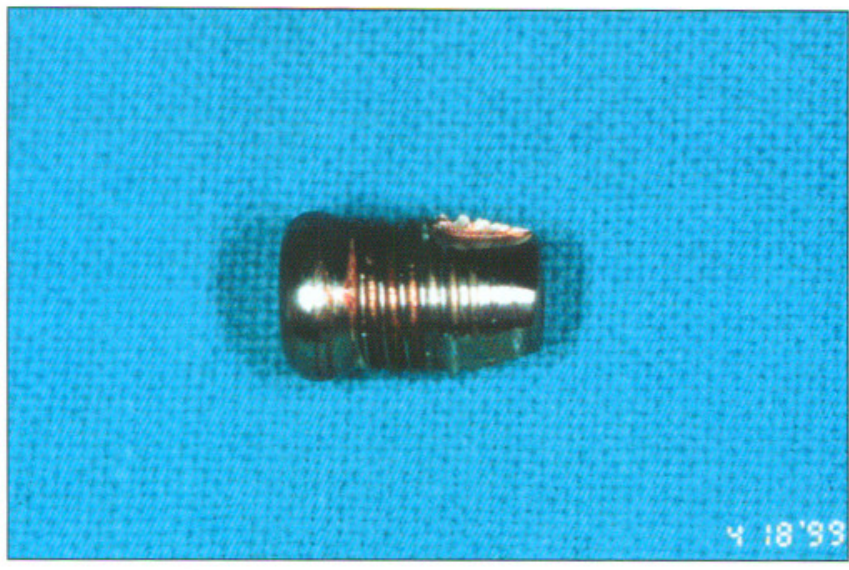

Fig. 6: Implante Brånemark Mk II $5 \varnothing \times 7 \mathrm{~mm}$ tras su remoción del seno maxilar.

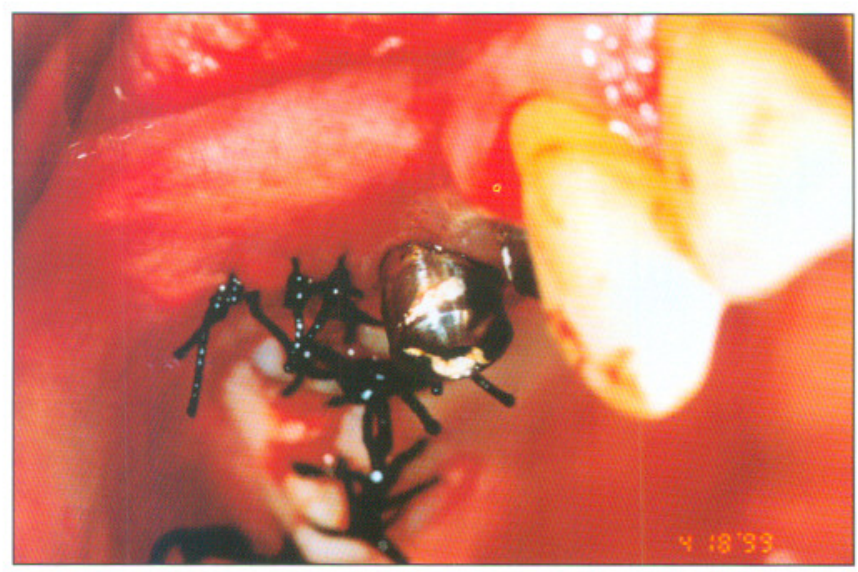

Fig. 8: Cierre completo de la herida quirúrgica mediante sutura con seda $3 / 0$.

se procedió a suturar con seda 3/0 (figura 8).

Durante el postoperatorio se indicó a la paciente la siguiente pauta farmacológica: Amoxicilina $500 \mathrm{mg}$ asociada al ácido clavulánico 125 mg (1 comprimido cada 8 horas durante 3 semanas), Diclofenaco $1 \mathrm{~g}$ cada 12 horas, Paracetamol $650 \mathrm{mg}$, y para prevenir una posible sinusitis, Loratadina $10 \mathrm{mg}$ cada 24 horas, Fenilefrina $500 \mathrm{mg}$ en espray cada 8 horas.

La herida quirúrgica cerró por primera intención y la paciente no presentó ninguna complicación postoperatoria, salvo una inflamación de la mejilla mayor que la esperada en una segunda fase de cirugía implantológica. Finalizado el periodo postoperatorio se procedió a la preparación de la prótesis fija después de considerar y rechazar la colocación de un nuevo implante en la misma localización.

Un año después fueron necesarias las extracciones de los dientes en posición 3.5, 3.6 y 3.7 y se colocaron tres nuevos implantes Brånemark $\mathrm{Mk}$ II en las mismas

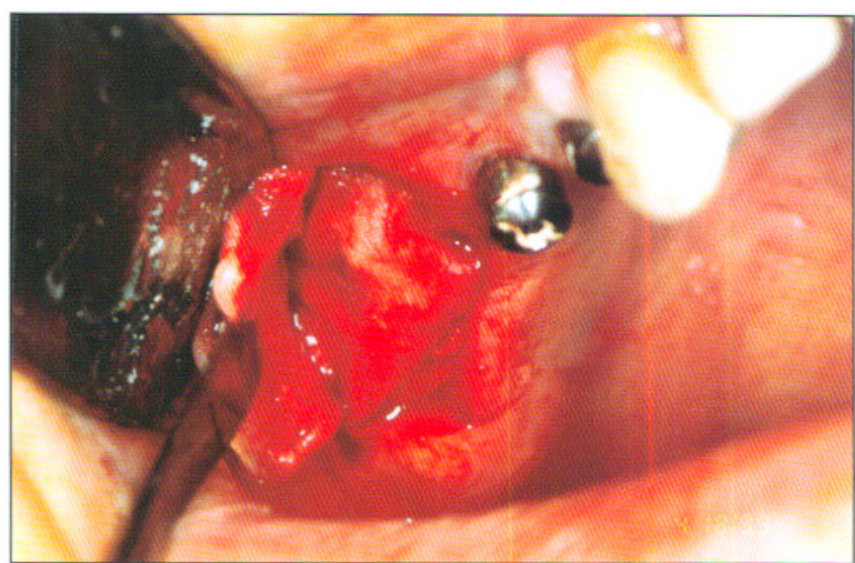

Fig. 7: Colocación de una membrana reabsorbible Bio-Guide ${ }^{\circ}$, en la zona del defecto óseo.

posiciones sin presentarse complicaciones postoperatorias.

El seguimiento de la paciente durante tres años muestra que los cinco implantes del maxilar superior y los tres implantes mandibulares pueden considerarse exitosos.

\section{DISCUSIÓN}

La colocación de implantes en zonas edéntulas posteriores del maxilar superior tiene importantes limitaciones anatómicas debido a una progresiva reabsorción del hueso alveolar remanente y a un incremento de la neumatización del seno maxilar, debiendo considerar además que en esta zona hay una calidad ósea deficiente para la colocación de implantes. Si con estas condiciones anatómicas y biológicas se realiza una inadecuada preparación del lecho implantario, con un fresado o instalación deficiente, todo ello puede conducir fácilmente a la aparición de complicaciones.

En este sentido, las complicaciones más frecuentes son la falta de estabilidad primaria del implante, la perforación de la cortical sinusal, una fenestración o dehiscencia de la cortical, una sinusitis postoperatoria, la creación de una comunicación bucosinusal y el fallo de la osteointegración. Otra posible complicación pero más infrecuente es el desplazamiento del implante hacia el seno maxilar.

Respecto a esta complicación que hemos descrito hay pocos casos referenciados en la literatura implantológica. Iida y cols. (6) publicaron un caso de un hombre que a la edad de 34 años se le reemplazó un segundo molar superior derecho con un implante. Cinco años más tarde, el paciente notó movilidad del 
implante y se le realizó una prótesis fija con un cantilever en la zona del implante no integrado sin incluirlo en la prótesis, pero éste no se eliminó. Diez años más tarde, una radiografía panorámica efectuada en una revisión en otro centro evidenció la penetración del implante en el suelo del seno maxilar; un año más tarde se observó el desplazamiento del implante en el interior del seno maxilar por lo que el paciente fue remitido al Departamento de Cirugía Oral y Maxilofacial de la Universidad de Osaka (Japón) donde le fue extraído el implante que finalmente se localizó en la pared medial del seno maxilar. La mucosa sinusal se dejó intacta ya que no mostraba infección ni cambios inflamatorios.

La penetración de un implante en el seno maxilar puede ocasionar serias complicaciones como sinusitis y la persistencia de una fístula bucoantral.

Regev y cols.(7) publicaron una serie de 8 pacientes con complicaciones sinusales en el tratamiento rehabilitador con prótesis implantosoportada. Uno de sus pacientes, un hombre con 61 años de edad que padecía una osteoporosis tratada con suplemento de calcio, fue intervenido para colocarle un injerto óseo autólogo en bloque tipo onlay de cresta ilíaca en la zona anterior del maxilar superior. Seis meses después se instalaron tres implantes tipo Mk II, uno cerca de la línea media y los otros dos en la zona correspondiente a los caninos. Durante la cirugía observaron que el hueso injertado era blando pero vital y estaba adherido al hueso primario del maxilar superior. Los implantes colocados se consideraron estables. Dos meses más tarde uno de los implantes fue expulsado a través de la nariz después de un estornudo según relató el paciente. La ortopantomografía evidenció que sólo uno de los implantes permanecía en su lecho quirúrgico, y además que el implante restante se había desplazado hacia el interior del seno maxilar derecho. Este implante fue extraído inmediatamente a través de una incisión de tipo Caldwell-Luc y en los controles posteriores no presentó infección sinusal ni fístula bucoantral. A este paciente se le realizó una sobredentadura utilizando el implante que permaneció integrado.

En otro de los casos publicados por Regev y cols.(7) de la misma serie, se trataba de una mujer de 66 años de edad con fibromiositis tratada con $5 \mathrm{mg}$ diarios de prednisona a la que se le colocaron dos implantes Brånemark de 10 y $7 \mathrm{~mm}$ en la zona posterior del maxilar superior izquierdo. Seis meses después se comprobó que sólo se integró el implante más anterior, y el otro tuvo que ser extraído para ser repuesto tras esperar tres meses. Este último implante perforó el suelo del seno maxilar sin producir sintomatología sinusal. Seis meses después de la colocación de este implante, durante el atornillado del pilar, como en nuestro caso, se produjo el desplazamiento de la fijación al interior del seno maxilar. Seguidamente fue retirado a través del lecho implantario labrado sin necesidad de efectuar la técnica de Caldwell-Luc.

Los mismos autores describen un tercer caso de desplazamiento de un implante. Se trataba del caso de un hombre de 34 años de edad con el antecedente de un traumatismo severo con fractura de la mandíbula y de varios dientes del maxilar superior. Durante la extracción de estos dientes se produjo una comunicación bucosinusal que cerró por primera intención. Ocho meses después del traumatismo se le realizó la técnica de elevación del seno y la colocación de tres implantes en el área premolar y molar derechos. Uno de los implantes no tuvo una estabilidad primaria adecuada ya que sólo existían $3 \mathrm{~mm}$ de altura de hueso alveolar remanente. A las dos semanas, una radiografía panorámica mostró el desplazamiento parcial y cambio de angulación del implante medial, pero a los 6 meses estaba integrado (7).

Quiney y cols.(8) han publicado el caso de una mujer de 44 años de edad con una historia de dos días de dolor facial unilateral y una importante tumefacción de la región malar izquierda. Dos semanas antes su dentista le colocó tres implantes en la zona alveolar superior izquierda. En la exploración observaron que la zona intervenida estaba eritematosa y tumefacta y el seno izquierdo estaba ocupado por un exudado purulento. Tras realizar una radiografía lateral de cráneo y una proyección de Waters se observó que el seno maxilar era radioopaco y que contenía dos implantes desplazados de su lecho quirúrgico. La ortopantomografía mostró un tercer implante localizado en la porción superior del seno maxilar. Los tres implantes fueron extraídos mediante una antrostomía sublabial con una extensa incisión en la mucosa vestibular. Realizaron el curetaje del hueso injertado y una antrostomía intranasal. También efectuaron un cultivo microbiológico que dio como resultado la presencia de un estreptococo hemolítico del grupo F y una mezcla de gérmenes anaerobios. El paciente siguió un tratamiento antibiótico estandar durante un mes y se recuperó con normalidad.

Ueda y Kaneda (9) publicaron dos casos de sinusitis crónica producida por el desplazamiento de implantes al seno maxilar. En el primer caso, una mujer de 36 años presentaba dolor facial unilateral e hinchazón en la región molar izquierda del maxilar superior. Dos meses antes le habían colocado tres implantes. En la exploración observaron que la encía de la zona intervenida estaba tumefacta, roja y blanda. La radiografía panorámica mostró la opacidad del seno maxilar izquierdo que contenía un implante no integrado además del tornillo de cierre. Tanto el implante como el tornillo de cierre fueron extraídos a través de una 
antrostomía sublabial efectuada con anestesia local y el hueso infectado se cureteó. Como tratamiento postoperatorio indicaron un mes de antibióticoterapia. El segundo caso es el de una mujer de 46 años de edad con dolor e inflamación de las dos mejillas. A esta paciente le habían colocado bilateralmente implantes cubiertos de "alumina ceramic", hacía 5 años. En la exploración se observó una severa inflamación y la formación de abscesos de partes blandas. En la radiografía panorámica se podía apreciar la extensa reabsorción ósea y el desplazamiento de los implantes a ambos senos maxilares. Los implantes fueron extraídos permaneciendo una fístula bucoantral durante 2 semanas después de la cirugía, que se cerró de forma espontánea tras el tratamiento antibiótico.

En nuestra opinión, el desplazamiento del implante hacia el interior del seno maxilar en el caso que hemos descrito en este artículo, se debe a una perforación de la cortical sinusal durante el fresado del lecho implantario durante la primera cirugía, que no es una complicación per se y a la no integración del implante. Estos dos factores favorecieron que cuando se intentó desatornillar el tornillo de cierre, se introdujera el implante en el seno maxilar por la presión ejercida durante la maniobra.

El desplazamiento de un implante hacia el interior del seno maxilar puede aparecer como una complicación en aquellos casos donde no hay estabilidad primaria debido a la escasa altura y la baja calidad ósea del hueso remanente o cuando el fresado ha sido incorrecto. Según otros autores (6) la existencia de una osteoporosis asociada a las fuerzas que recibe el hueso y que le son transmitidas a través de la prótesis, facilitan la movilidad del implante y su posible desplazamiento hacia el seno maxilar. Hay que tener en cuenta que cuando un implante penetra en el suelo del seno maxilar puede producir una reacción de cuerpo extraño y además los cambios de presión provocados en el seno pueden facilitar la migración de éste hacia el interior del mismo sin que existan unas fuerzas excesivas.

Sin embargo, Adell y cols.(10) publicaron una serie de 371 pacientes a quienes se había colocado implantes osteointegrados para su rehabilitación. Un total de 101 implantes colocados en el maxilar superior habían perforado el suelo del seno maxilar debido a que la altura del hueso alveolar era insuficiente y se buscaba su bicorticalización para ganar en estabilidad primaria, pero no se presentó ningún caso de sinusitis en un seguimiento de dos a diez años.

De todos modos, si nos encontramos en una situación de desplazamiento de un implante hacia el seno maxilar, lo indicado es su inmediata remoción, sin apelativos o de lo contrario aparecerá una sinusitis que agravará aún más el problema. Tras la extracción del implante debemos dar un tratamiento antiálgico, antibiótico, y descongestionantes nasales para prevenir la infección, y el exceso de inflamación y de secreciones durante el período de cicatrización.

Por otro lado, debemos considerar seriamente que para la colocación de implantes en la zona posterior del maxilar superior, donde la altura del hueso alveolar está comprometida y con una evidente baja calidad ósea, existen una serie de técnicas quirúrgicas que nos facilitan el éxito del tratamiento.

Una de las técnicas quirúrgicas más empleadas es la elevación del seno maxilar y su relleno con injerto de hueso autólogo o bovino o bien con materiales aloplásticos (11). Esta técnica se puede realizar simultáneamente a la colocación de los implantes o en dos fases quirúrgicas dependiendo de la altura del hueso alveolar residual existente. Es necesario un mínimo de 4 ó $5 \mathrm{~mm}$ para realizar la cirugía en una sola fase (12) que garantice la estabilidad primaria del implante. Sin embargo Peleg y cols.(13) efectuaron 20 elevaciones de seno maxilar en 20 pacientes simultáneamente a la colocación de un total de 55 implantes cubiertos de hidroxiapatita con una altura del hueso alveolar residual de 1 a $2 \mathrm{~mm}$, consiguiendo la integración de todos los implantes y la ausencia de complicaciones con una media de seguimiento de 26.4 meses. Estos autores proponen varias modificaciones en la técnica quirúrgica, como son una extensa ventana lateral, la utilización de hueso en partículas para homogeneizar el injerto óseo, una meticulosa condensación y buenas mediciones clínicas para asegurar el paralelismo de los implantes.

Dentro de esta modalidad quirúrgica, la utilización de osteotomos permite llevar a cabo una técnica más atraumática que la elevación sinusal clásica por vía vestibular. En este sentido, Rosen y cols.(14) realizaron un estudio multicéntrico retrospectivo de la técnica de elevación sinusal mediante osteotomos. El éxito global de supervivencia de los implantes fue del $95,4 \%$ con un seguimiento medio de carga de 20,2 meses y un rango entre 6 y 66 meses. Sin embargo, cabe destacar que se utilizaron implantes de diferentes marcas comerciales, con distinto tratamiento de superficie, de diseño cilíndrico y roscado, y que los 9 cirujanos que efectuaron el estudio usaron materiales de relleno en distintas cantidades y proporciones según el criterio de cada uno de ellos, hechos que reducen la fiabilidad del trabajo. Los autores concluyen afirmando que esta técnica es buena utilizando varios tipos de implantes y materiales de relleno, y que el factor más importante en la supervivencia del implante es la distancia entre el suelo del seno maxilar y la cresta alveolar, ya que si ésta es inferior a 4 milímetros la supervivencia de las fijaciones es del 
$85,7 \%$ y si es igual o superior a 5 milímetros, este mismo porcentaje aumenta por encima del $96 \%$.

En el maxilar superior Kremanov y cols.(15) proponen que los implantes sean paralelos a las paredes anterior y posterior del seno maxilar, así como también perpendiculares a la curvatura palatina y recomiendan la ubicación de algún implante en la apófisis pterigoides. Para comprobar su filosofía de trabajo realizaron un estudio con un total de 47 pacientes. A 25 de ellos les colocaron 86 implantes en la mandíbula y a los 22 restantes 75 implantes en el maxilar superior. Defienden esta opción terapéutica porque creen que así se evita efectuar técnicas quirúrgicas más agresivas y con una mayor morbilidad; se pueden colocar implantes más largos con lo que se consigue un mayor éxito en la osteointegración; se puede ofrecer al paciente una mejor oclusión en los sectores posteriores además de reducir la longitud del cantilever en la prótesis, aunque se deberá compensar la angulación de los implantes con la utilización de pilares de cicatrización angulados y, finalmente, se consigue aumentar la estabilidad primaria de los implantes.

Una técnica más agresiva pero indicada en pacientes edéntulos con reabsorción severa del maxilar superior es la osteotomía de Le Fort I y posterior rehabilitación con implantes. Esta cirugía está indicada en pacientes con una distancia intermaxilar aumentada por la reabsorción y con una discrepancia sagital que hace que los maxilares se encuentren en clase III esquelética. El éxito de osteointegración en estos casos es del $94,6 \%$ en el grupo de pacientes operados por Stoelinga y cols. (16). Estos mismos autores refieren que esta técnica tiene una gran ventaja frente a los onlays ya que se conserva encía queratinizada alrededor de las fijaciones, y no crea la necesidad de realizar injertos de encía libre. Sin embargo, en ocasiones es difícil hacer una osteotomía controlada del maxilar superior y se pueden fracturar las tuberosidades o el hueso palatino. Además, al descender el maxilar superior se reduce el espacio que ocupa la lengua y el paciente puede tener problemas foniátricos.

Una de las dudas que se plantea en el tratamiento de las reabsorciones óseas severas es si es mejor colocar los implantes en la misma cirugía de aumento o bien que se realice una técnica diferida (12, 13). En el estudio de Wannfors y cols.(17) en 40 pacientes con el maxilar superior edéntulo, realizaron la técnica simultánea en 20 pacientes y la técnica diferida en los otros 20. Concluyeron que el riesgo de pérdida de los implantes en una sola cirugía es dos veces mayor que en la técnica diferida asegurando, con esta última, mejores resultados y consiguiendo disminuir la frecuencia de complicaciones.

\section{SUIMIMARY}

The oral rehabilitation of the patients with partial or total edentulism with the use of dental implants has wide applications. In occasions, these applications are limited by anatomical and biological factors. Concretely, the posterior area of the maxilla can have a scarce height of residual ridge, besides a poor bony quality and further pneumatization of the maxillary sinus. All this makes that so much the conventional surgical technique as other alternative procedures as sinus lift, onlay graft, the technique with osteotoms and the osteotomy of Le Fort I, be surgical procedures that can change the anatomy and physiology of the maxillary sinus favoring the appearance of complications. One of the possible but rare complications is the displacement of the dental implants toward the maxillary sinus as the case that we describe in this article.

\section{KEY WORDS}

Dental implant, maxillary sinus, displacement, complications.

\section{CORRESPONDENCIA}

Dirección: Dr. Cosme Gay Escoda.

C/ Ganduxer 140, 4․

08022 Barcelona.

E-mail: cgay@bell.ub.es.

http://www.gayescoda.com

\section{BIBLIOGRAFÍA}

1. Sánchez MA, Alaejos C, Valmaseda E, Contreras MA, Abad D, Berini L, Gay-Escoda C. Revisión de los artículos de implantología bucal publicados durante 1993 y 1994 Arch Odontoestomatol 1996; 12: 459-69.

2. Balshi T. Commentary and Analysis. Impl Dentistry 2000; 9: 18-9.

3. Raghoebar GM, Vissink A, Reintsema H, Batenburg HK. Bone grafting of the floor of the maxillary sinus for the placement of endosseous implants. Br J Oral Maxillofac Surg 1997; 35:119-25.

4. Jensen J, Sindent-Petersen S, Olivier AJ. Varying treatment strategies for reconstruction of maxillary atrophy with implants: results in 98 patients. J Oral Maxillofac Surg 1994; 52: 210-6. 


\section{AVANCES}

Volumen 14 - $\mathrm{N}^{\circ} 2$ - Julio 2002

5. Chanavaz M. Maxillary sinus: anatomy, physiology, surgery and bone grafting relating to implantology. Eleven years experience (1979-1990). J Oral Implantol 1990;16: $199-209$.

6. Iida S, Tanaka N, Kogo M, Matsuya T. Migration of a dental implant into the maxillary sinus. A case report. Int J Oral Maxillofac Surg 2000; 29: 358-9.

7. Regev E, Smith RA, Perrott DH, Pogrel MA. Maxillary sinus complications related to endosseous implants. Int J Oral Maxillofac Implants 1995; 10: 451-61.

8. Quiney RE, Brimble E, Hodge M. Maxillary sinusitis from dental osseointegrated implants. J Laryngol Otol 1990; 104: 333-4.

9. Ueda M, Kaneda T. Maxillary sinusitis caused by dental implants: report of two cases. J Oral Maxillofac Surg 1992; 50: 285-7.

10. Adell R, Lekholm U, Rockler B, Branemark PI. A 15 year study of osseointegrated implants in the treatment of the edentulous jaw. Int J Oral Surg 1981; 10:387-416.

11. Guerrero A, Lundgren S, Salinas JL. Desplazamiento óseo del suelo del seno maxilar con injerto autógeno particulado tomado de localizaciones mandibulares: descripción de la técnica. Arch Odontoestomatol 1997; 13: 3-13.

12. Peleg M, Mazor Z, Garg AK. Augmentation grafting of the maxillary sinus and simultaneous implant placement in patients with 3 to $5 \mathrm{~mm}$ of residual alveolar bone height. Int J Oral Maxillofac Implants 1999; 14:549-56.
13. Peleg M, Mazor Z, Chaushu G, Garg AK. Sinus floor augmentation with simultaneous implant placement in the severely atrophic maxilla. J Periodontol 1998; 69:1397403.

14. Rosen PS, Summers R, Mellado JR, Salkin LM, Shanaman $\mathrm{RH}$, Marks $\mathrm{MH}$ et al. The bone-added osteotome sinus floor elevation technique: multicenter retrospective report of consecutively treated patients. Int J Oral Maxillofac Implants 1999;14:853-8.

15. Kremanov L, Kahn M, Rangert B, Lindström H. Tilting of posterior mandibular and maxillary implants for improved prosthesis support. Int J Oral Maxillofac Implants 2000; 15: 405-14.

16. Stoelinga PJ, Slagter AP, Brouns JJA. Rehabilitation of patients with severe (class VI9 maxillary resorption using Le Fort I osteotomy, interposed bone grafts and endosteal implants: 1-8 years follow-up on a two-stage procedure. Int J Oral Maxillofac Surg 2000;29:188-93.

17. Wannfors K, Johansson B, Hallman M, Strandkvist T. A prospective randomized study of 1 - and 2-stage sinus inlay bone grafts: 1-year follow-up. Int J Oral Maxillofac Implants 2000;15:625-32. 